\title{
MODIFICAÇÕES NOS INDICADORES SOCIAIS DA REGIÃO NORDESTE APÓS A IMPLEMENTAÇÃO DA ATENÇÃO PRIMÁRIA
}

\author{
CHANGES IN SOCIAL INDICATORS OF THE NORTHEASTERN REGION \\ AFTER THE IMPLEMENTATION OF PRIMARY HEALTH CARE
}

Fátima Cristina Dias de Carvalho (iD) ${ }_{(0000-0002-2790-6414)^{1}}$, Thiago Brasileiro de Vasconcelos (iD) (00000002-9554-1736) ${ }^{2}$, Gisele Maria Melo Soares Arruda ${ }_{(0000-0002-8815-7955)}^{3}$, Raimunda Hermelinda Maia Macena ${ }_{(0000-0002-3320-8380)^{2}}$

\footnotetext{
${ }^{1}$ Universidade Federal do Ceará, Departamento de Fisioterapia, Fortaleza, Ceará, Brasil.

${ }^{2}$ Centro Universitário Católica de Quixadá, Curso de Fisioterapia, Quixadá, Ceará, Brasil. $<$ thiagobvasconcelos@hotmail.com>

${ }^{3}$ Universidade Estadual do Ceará, Curso de Medicina, Fortaleza, Ceará, Brasil.
}

Resumo O objetivo foi descrever a implementação da Atenção Primária à Saúde no Nordeste brasileiro e sua correlação com os indicadores sociais. Trata-se de estudo ecológico com dados do Departamento de Atenção Básica do Ministério da Saúde e Instituto Brasileiro de Geografia e Estatística, tendo como variável de estudo o número de Agentes Comunitário de Saúde, Equipes de Saúde da Família e Núcleos de Apoio à Saúde da Família. O cálculo da tendência temporal de Agentes Comunitários de Saúde e Equipes de Saúde da Família foi realizado por regressão joinpoint de Poisson. Foi observada correlação forte $(r \geq 0,7)$ entre os indicadores sociais e a implantação da Atenção Primária à Saúde e do Núcleo de Saúde da Família na região Nordeste, esgotamento sanitário ina-dequado, taxa de fecundidade total, domicílio com água encanada, percentual de domicílios com coleta de lixo, esperança de vida ao nascer, taxa de envelhecimento e probabilidade de sobreviver até 60 anos. As variáveis de desenvolvimento e de saúde apresentaram modificação no coeficiente de correlação e de determinação, com significância estatística ( $\mathrm{p}<$ $0,05)$. Há uma tendência temporal de crescimento na implantação da Atenção Primária à Saúde no Nordeste, bem como a existência de forte correlação entre os indicadores sociais e de saúde.

Palavras-chave avaliação em saúde; Atenção Primária à Saúde; Estratégia Saúde da Família; indicadores sociais; saúde pública.
Abstract The goal of the present study was to describe the implementation of Primary Health Care in the Northeastern Region of Brazil and its correlation with social indicators. It is an ecological study with data from the Primary Health Care Department (Departamento de Atenção Básica, in Portuguese) of the Brazilian Ministry of Health and from the Brazilian Institute of Geography and Statistics (Instituto Brasileiro de Geografia e Estatística, in Portuguese), and the variable of the study is the number of Community Health Workers, Health of the Family Teams and Health of the Family Support Centers. The calculation of the time trend of the Community Health Workers and of the Health of the Family Teams was performed using Poisson's joinpoint regression. We observed a strong correlation $(r \geq 0.7)$ between the social indicators and the implementation of Primary Health Care and of the Health of the Family Center in the Northeastern Region, inadequate sanitary sewage systems, total fertility rate, houses with indoor plumbing, percentage of houses with garbage collection, life expectancy at birth, aging rate, and probability of living until the age of 60 years. The health and development variables presented a statistically significant ( $p$ $<0.05)$ change in the correlation and determination coefficient. There is a time trend regarding the increase in the implementation of Primary Health Care in the Northeast, and there is also a strong correlation between the social and health indicators.

Keywords evaluation in health; Primary Health Care; Family Health Strategy; social indicators; public health. 


\section{Introdução}

A Atenção Primária à Saúde (APS) foi proposta para a organização de serviços com foco nas necessidades de saúde da população adscrita e baseada em uma ação multiprofissional e interdisciplinar com participação social. Na organização do modelo assistencial em saúde brasileiro - o Sistema Único de Saúde (SUS) -, a APS assume o papel de ordenadora do cuidado dentro de um sistema poliárquico, sendo denominada, à época de sua implantação no país, como Atenção Básica (AB) para enfatizar a reorientação da assistência em saúde segundo um sistema universal e integrado e diferenciar-se das iniciativas focalizadas e seletivas que estavam sendo implantadas em outras realidades (Matta e Morosini, 2009; Mendes, 2011; Giovanella e Mendonça, 2012).

A Atenção Básica surgiu com a reforma sanitária brasileira, caracterizavase por serviços ambulatoriais básicos ofertados mais próximo da realidade de vida da população. Esses serviços ampliaram o acesso, todavia não se tratava de uma estratégia sistematizada e pautada na clínica ampliada. Para a implementação da Atenção Básica brasileira, foram desenvolvidas algumas experiências exitosas que culminaram com a reorganização da APS brasileira. Como exemplo, pode-se citar a criação dos Agentes Comunitários de Saúde (ACSs), no final dos anos 1980, tanto em uma experiência direcionada pela Universidade de Brasília em Planaltina-DF quanto no sertão cearense para o combate à mortalidade infantil (Ávila, 2011).

Os ACSs, até então coordenados por um profissional de enfermagem, passaram, em alguns municípios do Ceará, desde 1994, a fazerem parte de uma equipe ampliada pela presença do profissional médico. Criava-se, em 1994, a Equipe de Saúde da Família (EqSF) que culminou, então, com a regulamentação nacional de dois programas: Programa de Saúde da Família (PSF) e o Programa de Agentes Comunitários de Saúde (PACS) em 1997. O PSF, criado em 1994 e regulamentado em 1997, foi entendido como um programa de transição para a implantação da Estratégia de Saúde da Família (ESF), que surgiu, em 2006, como iniciativa para reorientar nacionalmente o modelo de atenção à saúde com base na APS com vistas a auxiliar na melhoria dos indicadores sociais (Figueiredo, 2012). A APS é considerada como porta de entrada preferencial ao SUS e atua em uma complexidade do cuidado das famílias cabendo-lhe a indissociabilidade entre ações curativas e preventivas. No ano de 2008, o Ministério da Saúde cria os Núcleos de Apoio à Saúde da Família (NASFs) com o intuito de ampliar o escopo e a abrangência das ações da APS além de servir como apoio matricial às EqSFs (Brasil, 2017). Atualmente, a ESF constitui-se como o modelo predominante de estruturação da APS brasileira, todavia, os autores deste artigo compreendem como Atenção Básica todas as iniciativas de implementação da APS, mesmo aquelas anteriores ao PSF. 
Evidências na literatura mostram que países que adotam o modelo de APS como base do sistema de saúde desenvolvem ações que se associam positiva e significativamente, com incremento em indicadores sociais, melhora da qualidade de vida da comunidade, elevação das taxas de detecção de doenças crônicas, redução da mortalidade precoce devido às causas preveníveis, diminuição da demanda excessiva no nível de maior densidade tecnológica (Malta et al., 2016; Moretti e Fedosse, 2016).

Deste modo, preconiza-se que os serviços de saúde oferecidos no SUS sejam organizados em uma rede de cuidados articulada, com controle dos fluxos, objetivando atender as necessidades da população com vistas à melhoria da qualidade de vida e bem-estar, bem como propiciar um modelo de gestão baseado na vigilância em saúde (Oliveira e Pereira, 2013). A prática da Vigilância em Saúde, na APS, objetiva sistematizar os problemas de saúde por meio de indicadores que permitem identificar e monitorar as necessidades de saúde, o bem-estar da população, sendo norteador do gerenciamento, avaliação e planejamento das ações em saúde, favorecendo a busca de melhorias nos processos e resultados das ações de saúde (Sumar e Fausto, 2014; Akerman e Furtado, 2017).

Apesar da expressiva expansão da ESF em todo o território brasileiro nos últimos anos, o processo de implantação ocorreu em diferente velocidade e intensidade nas diversas regiões do país. Ainda é díspar a implantação da ESF no país. Em 2012, por exemplo, 95\% dos municípios brasileiros contavam com um total de 33.404 EqSFs implantadas, com potencial para abranger apenas $55 \%$ da população brasileira. Embora com elevada proporção de domicílios cadastrados, a região Nordeste destacava-se, significativamente, quanto à vinculação de famílias da zona rural em detrimento da zona urbana (Malta et al., 2016).

Entretanto, ainda é incerta a relação entre a implementação da APS na região do Nordeste brasileiro e suas modificações nos indicadores sociais. Diante disto, este estudo visa descrever a implementação da $\mathrm{AB}$ no Nordeste brasileiro e sua correlação com os indicadores sociais.

\section{Métodos}

Foi realizado um estudo ecológico, descritivo por meio de análise de série temporal para avaliação do processo de implementação da APS no Nordeste brasileiro, tendo como unidade para análise os anos de 1998 a 2016.

A região Nordeste é composta por nove estados: Alagoas, Bahia, Ceará, Maranhão, Paraíba, Pernambuco, Piauí, Rio Grande do Norte e Sergipe. Considerada a maior região em número de estados, possui uma área estimada de $1.561 .177,8 \mathrm{~km}^{2}$, equivalente a $18,3 \%$ do território nacional, com 53,1 
milhões de habitantes, sendo a segunda maior população no país (Francisco, 2017).

Foram usados dados secundários oriundos do Departamento de Atenção Básica (DAB) e Instituto Brasileiro de Geografia e Estatística (IBGE), de domínio público e disponibilizados on-line, tendo como variável de estudo o número de ACSs, EqSFs e NASFs credenciados, cadastrados e implementados no período de estudo. Optou-se por analisar de 1998 a 2016, pois os dados disponíveis no DAB constam desde 1998.

Os dados de implantação da atenção básica foram obtidos no $\mathrm{DAB}^{1}$, com base na competência por Unidade Geográfica para os períodos de dezembro de 1998 a dezembro de 2016. Foram usados campos relativos a quantidade de municípios com ACS e EqSF; quantidade de ACSs, EqSFs e NASFs (1, 2 e 3) credenciados, cadastrados e implantados. Foi criada a variável implantação da AB com base na informação do município ter ACS, EqSF e/ou NASF implantado.

Os dados populacionais foram obtidos no IBGE, disponíveis pela internet ${ }^{2}$, com base nos Censos Nacionais de População (2000 e 2010) e nas estimativas de população e indicadores sociais para os anos intercensitários (2001-2009 e 2011-2016).

A mensuração do estado de saúde da população reflete sobre condições de vida, acesso a serviços de saúde e qualidade da atenção primária. No Brasil, o grupo de Indicadores e Dados Básicos (IDB) reúne um vasto conjunto de dados demográficos, socioeconômicos, de morbimortalidade e fatores de risco, além de estatísticas sobre recursos e cobertura de serviços. Esses indicadores estão relacionados a diversos aspectos determinantes do estado de saúde do país e de diferentes grupos populacionais (Brevidelli e Freitas, 2011). Deste modo, as variáveis dependentes estudadas foram nomeadas como indicadores sociais de desenvolvimento e de saúde.

Os indicadores sociais de desenvolvimento foram: o 'Índice de Gini' que mede o grau de desigualdade existente na distribuição de indivíduos ${ }^{3}$ segundo a renda domiciliar per capita (varia de 0 , quando não há desigualdade, à 1, quando a desigualdade é máxima); o percentual da população que vive em domicílios com água encanada (razão entre a população que vive em domicílios particulares permanentes com água canalizada ${ }^{4}$ para um ou mais cômodos e a população total residente em domicílios particulares permanentes multiplicados por 100); o percentual da população que vive em domicílios urbanos com serviço de coleta de lixo (razão entre a população que vive em domicílios com coleta de lixo $0^{5}$ e a população total residente em domicílios particulares permanentes multiplicados por 100); o percentual de pessoas em domicílios com abastecimento de água e esgotamento sanitário inadequados (razão entre as pessoas que vivem em domicílios cujo abastecimento de água não provém de rede geral e cujo esgotamento sanitário não é realizado por rede coletora de esgoto ou fossa séptica e a população total residente em do- 
micílios particulares permanentes multiplicado por 100); o 'IDHM - Índice de Desenvolvimento Humano Municipal (corresponde à média geométrica dos índices das dimensões Renda, Educação e Longevidade, com pesos iguais) e o 'Índice de Desenvolvimento Humano Municipal - Dimensão Longevidade (IDHM Longevidade)' obtido com base no indicador Esperança de vida ao nascer. ${ }^{6}$ Para os indicadores relacionados a domicilio foram considerados apenas os domicílios particulares permanentes.

Foram considerados indicadores sociais de saúde: 'taxa de fecundidade total' - número médio de filhos que uma mulher deverá ter ao terminar o período reprodutivo (15 a 49 anos de idade); 'esperança de vida ao nascer' - número médio de anos que as pessoas deverão viver desde o nascimento, se permanecerem constantes ao longo da vida o nível e o padrão de mortalidade por idade prevalecente no ano do Censo; 'percentual de mulheres jovens com filhos' - razão entre a população de mulheres de 10 a 17 anos que tiveram filhos e a população total de mulheres de 10 a 17 anos residente na região multiplicados por $100 \%$; 'mortalidade infantil' - número de crianças que não deverão sobreviver ao primeiro ano de vida em cada 1000 nascidos vivos; 'mortalidade até 5 anos de idade' - probabilidade de morrer entre o nascimento e a idade exata de 5 anos, por 1000 nascidos vivos; 'taxa de envelhecimento' - razão entre a população de 65 anos ou mais de idade e a população total multiplicado por 100 e 'probabilidade de sobrevivência até 60 anos' - probabilidade de uma criança recém-nascida viver até os 60 anos, se permanecerem constantes ao longo da vida o nível e o padrão de mortalidade por idade do ano do Censo.

Os dados foram exportados para o software Microsoft Office Excel ${ }^{\circledR}$ for Windows 2010 e as análises estatísticas realizadas com o software Stata ${ }^{\circledR}$ versão 11.2 (StataCorp LP, CollegeStation, TX, EUA). Foi calculada a estatística descritiva e analisado o coeficiente de correlação e de determinação dos indicadores sociais e a implantação da Atenção Básica, ACS, EqSF e NASF, considerando a significância estatística p < 0,05 (5\%).

Para o cálculo da tendência temporal de ACS e EqSF foi aplicada a regressão Joinpoint de Poisson (por pontos de inflexão) empregando-se o Joinpoint Regression Program versão 4.4.2). Com base na definição dos seguimentos, estimou-se e testou-se a Variação Percentual Anual (Anual Percentual Change - APC) e a Variação Percentual Anual Média (Average Anual Percentual Change - AAPC), com seus respectivos intervalos de confiança (IC) de 95\%. O resultado possibilitou demonstrar crescimento (valores de APC positivos), redução (valores de APC negativos) ou manutenção (valor de APC igual a zero) da tendência ao longo de toda a série histórica que foi analisada.

O presente estudo usou dados de acesso público, cujas informações são agregadas, sem possibilidade de identificação individual. Desta forma não foi 
necessário submeter o projeto deste trabalho para apreciação de um Comitê de Ética em Pesquisa (CEP) (Brasil, 2017b).

\section{Resultados}

No ano de 1998, 78\% dos municípios da região Nordeste possuíam ACSs, no mesmo período apenas $22 \%$ dos municípios apresentavam EqSFs e não existia NASF. Observa-se que o número de municípios que aderiram ao processo de implantação de ACSs e EqSFs apresenta crescimento até 2003 e 2008, respectivamente. O número de municípios com ACSs implantados teve queda discreta no ano de 2004, voltando a crescer e, desde então, observou-se uma tendência à estabilização. Entretanto, mesmo neste período depois de 2005 em que se estabilizou o número de municípios com ACSs, a cobertura populacional de ACSs passou de $56,56 \%$ para $86,86 \%$, já o número de EqSFs teve um aumento significativo de 9,32\% para 79,42\%. Ou seja, houve um aumento na quantidade absoluta de EqSFs mesmo que não tenha aumentado o número de municípios que aderiram à ESF. Somente em 2010, os municípios do Nordeste procederam à implantação do NASF, totalizando em 33\% destes municípios com NASF; nesse mesmo período $100 \%$ já possuíam ACSs e EqSFs. Em seis anos, o número de municípios do Nordeste com NASF triplicou (Gráfico 1). 


\section{Gráfico 1}

Implantação municipal da Atenção Básica (ACS, EqSF e NASF) nos municípios do Nordeste brasileiro (1998-2016). Fortaleza/CE, 2017.

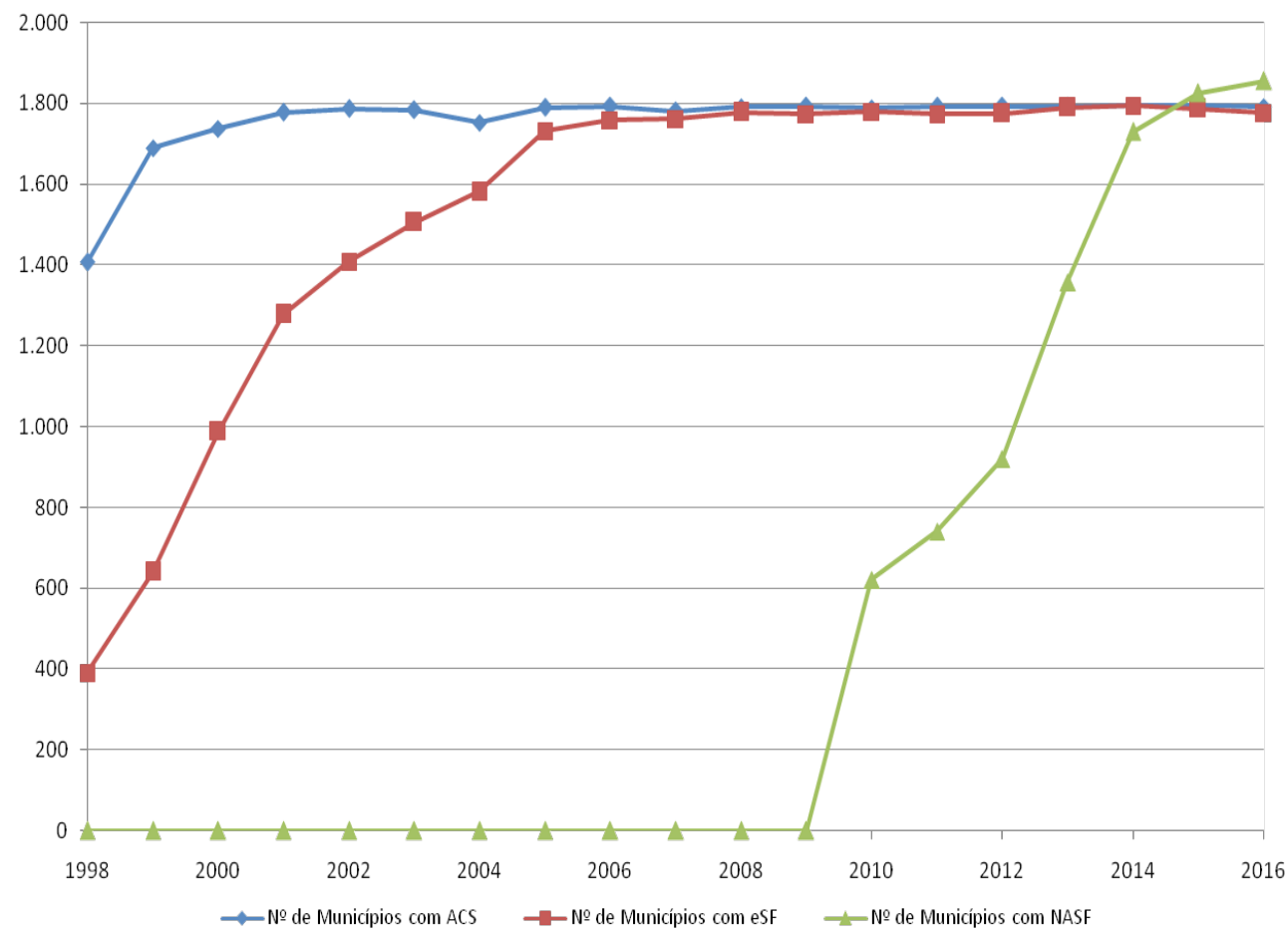

Nota: $\mathrm{ACS}=$ Agente Comunitário de Saúde; EqSF = Equipes de Saúde da Família e NASF = Núcleos de Apoio à Saúde da Família.

No Nordeste do Brasil, entre 1998 e 2016, observou-se que o número médio de equipes de $\mathrm{AB}$ credenciadas foi de $111.576 \mathrm{DP}: 26.522$, sendo 12,33\% maior que o de equipes cadastradas (média $=97.818$ DP: 21.700 ) e 13,61\% maior que as efetivamente implantadas (média $=96.390$ DP: 21.233), o que acarretou no período uma relação média de 552 habitantes para cada equipe de $\mathrm{AB}$ com cobertura populacional de apenas $21,26 \%$ na região (Tabela 1). 
Tabela 1

Dados descritivos da implantação de Atenção Básica, ACS, EqSF e NASF no Nordeste brasileiro período de 19982016. Fortaleza, CE, 2017.

\begin{tabular}{|c|c|c|c|c|}
\hline Variáveis & MIN & MAX & MÉDIA & DP \\
\hline \multicolumn{5}{|l|}{ AB } \\
\hline N. de credenciados & 52.483 & 136.962 & 111.576 & 26.522 \\
\hline N. de cadastrados & 49.131 & 120.570 & 97.818 & 21.700 \\
\hline N. de implantados & 48.611 & 118.714 & 96.390 & 21.233 \\
\hline Implantado/cadastrado & 0,98 & 0,99 & 0,99 & 0,00 \\
\hline Implantado/credenciado & 0,80 & 0,97 & 0,87 & 0,04 \\
\hline N. habitantes/AB & 454 & 933 & 552 & 124 \\
\hline Cobertura populacional (\%) & 10,72 & 26,19 & 21,26 & 4,68 \\
\hline \multicolumn{5}{|l|}{ ACS } \\
\hline Cobertura municípios (\%) & 78,42 & 100,00 & 98,08 & 4,98 \\
\hline N. de credenciados & 51.120 & 117.292 & 98.526 & 20.878 \\
\hline N. de cadastrados & 47.894 & 105.222 & 87.686 & 17.221 \\
\hline N. de implantados & 47.381 & 103.637 & 86.417 & 16.845 \\
\hline Implantado/cadastrado & 0,98 & 0,99 & 0,99 & 0,00 \\
\hline Implantado/credenciado & 0,81 & 0,97 & 0,88 & 0,04 \\
\hline N. habitantes/ACS & 520,15 & 956,81 & 608,17 & 111,59 \\
\hline \multicolumn{5}{|l|}{ EqSF } \\
\hline Cobertura municípios (\%) & 21,81 & 100,00 & 85,32 & 23,43 \\
\hline N. de credenciados & 1.363 & 17.638 & 12.499 & 5.162 \\
\hline N. de cadastrados & 1.237 & 15.362 & 10.082 & 4.472 \\
\hline N. de implantados & 1.230 & 15.065 & 9.925 & 4.385 \\
\hline Implantado/cadastrado & 0,97 & 1,00 & 0,98 & 0,01 \\
\hline Implantado/credenciado & 0,68 & 0,90 & 0,79 & 0,06 \\
\hline N. habitantes/EqSF & 3.578 & 36.857 & 8.082 & 8.635 \\
\hline \multicolumn{5}{|l|}{ NASF $(1+2+3)$} \\
\hline N. de credenciados & 0 & 2.032 & 551 & 812 \\
\hline N. de cadastrados & 0 & 272 & 49 & 101 \\
\hline N. de implantados & 0 & 263 & 49 & 99 \\
\hline Implantado/cadastrado & 0,96 & 1,00 & 0,99 & 0,02 \\
\hline Implantado/credenciado & 0,09 & 0,13 & 0,12 & 0,02 \\
\hline N. habitantes/NASF & 0 & 86.310 & 18.188 & 27.832 \\
\hline \multicolumn{5}{|l|}{ NASF 1} \\
\hline N. de credenciados & 0 & 1.358 & 388 & 551 \\
\hline N. de cadastrados & 0 & 1.259 & 344 & 491 \\
\hline N. de implantados & 0 & 1.214 & 338 & 480 \\
\hline Implantado/cadastrado & 0,96 & 1,00 & 0,98 & 0,01 \\
\hline
\end{tabular}




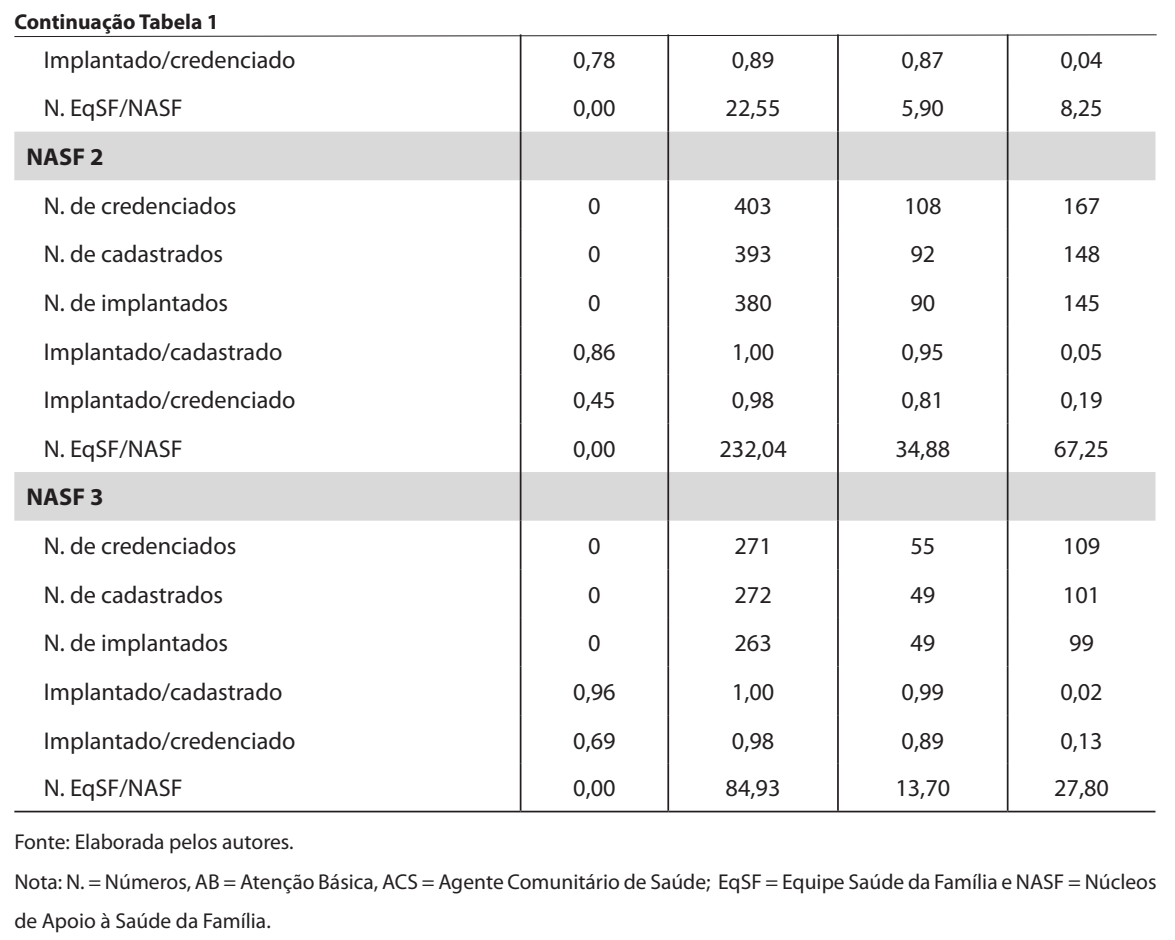

Observou-se o número médio de ACS credenciados de 98.526 DP: 20.878, $10,81 \%$ maior que os cadastrados (87.686 DP: 17.221) e 12,29\% maior que os implantados (86.417 DP: 16.845), acarretando no período (1998-2016) uma relação média de 608,17 habitantes por ACS. O número médio de EqSFs credenciadas foi de 12.499 DP: 5.162, representando um percentual de 19,33\% maior que as cadastradas (10.082 DP: 4.472) e 20,59\% maior que as implantadas (9.925 DP: 4.385), o que acarretou no período uma relação média de 8.082 habitantes por cada EqSF (Tabela 1).

Em relação a todos os tipos de NASF, observou-se que o número médio de credenciados foi de 551 DP: 812, 91,1\% maior que os cadastrados (49 DP: 101) e os implantados (49 DP: 99), com uma relação média de 18.188 habitantes para cada NASF. Ao se especificar por cada tipo de NASF, observou-se que o número médio de NASFs 1 credenciados foi de 388 DP: 551, 34\% maior que os cadastrados (344 DP: 491) e 76,80\% que o efetivamente implantados (338 DP: 480), com um número médio de 5,30 EqSFs por NASFs 1; já para os NASFs 2 observou-se número médio de credenciados = 108 DP: 167, 14,81\% maior que os cadastrados (92 DP: 148) e 16,66\% que os implantados (90 DP: 145), com número médio de 34,88 EqSFs por NASFs 2; e ao analisar os NASFs 3 observou-se que os credenciados apresentaram média de 55 DP: 109, o que representa 10,90\% dos cadastrados (49 DP: 101), correspondendo à mesma porcentagem dos implantados (49 DP: 99) com número médio de 13,70 EqSFs por NASFs 3 (Tabela 1). 
Foi observada correlação forte entre os diversos indicadores sociais estudados e a implantação da $\mathrm{AB}$ e do NASF, na região Nordeste, ao longo dos anos. Enquanto o índice de Gini $(r=-0,73)$, o esgotamento sanitário inadequado $(\mathrm{r}=-0,70)$, a taxa de fecundidade total $(\mathrm{r}=-0,71)$, o percentual de mulheres jovens com filhos $(r=-0,72)$, a mortalidade infantil $(r=-0,66)$, e a mortalidade até 05 anos $(r=-0,64)$ obtiveram correlação forte e negativa; os indicadores domicílio com água encanada $(\mathrm{r}=0,78)$, percentual domicílio com coleta de lixo $(r=0,76)$, IDHM $(r=0,77)$, IDHM Longevidade $(r=0,75)$, esperança de vida ao nascer $(r=0,75)$, taxa de envelhecimento $(r=0,76)$ e probabilidade de sobreviver até 60 anos $(r=0,74)$ obtiveram correlação forte e positiva. Entretanto, a correlação entre os indicadores sociais e NASF foi menor que entre ACS, EqSF e AB de modo geral (Tabela 2).

Tabela 2

Correlação e determinação dos indicadores sociais e a implantação da Atenção Básica, ACS, EqSF e NASF no Nordeste brasileiro (1998-2016). Fortaleza, CE, 2017.

\begin{tabular}{|c|c|c|c|c|c|c|c|c|c|c|c|c|}
\hline \multirow{2}{*}{ Variáveis } & & \multicolumn{3}{|c|}{ ACS } & \multicolumn{3}{|c|}{ EqSF } & \multicolumn{3}{|c|}{ NASF } \\
\hline & $\mathbf{r}$ & valor-P & $\mathbf{r}^{2}$ & $\mathbf{r}$ & valor-P & $\mathbf{r}^{2}$ & $\mathbf{r}$ & valor-P & $\mathbf{r}^{2}$ & $\mathbf{R}$ & valor-P & $\mathbf{r}^{2}$ \\
\hline \multicolumn{13}{|c|}{ Desenvolvimento } \\
\hline Índice de Gini & $-0,96$ & 0,000 & 0,93 & $-0,96$ & 0,000 & 0,92 & $-0,96$ & 0,000 & 0,93 & $-0,73$ & 0,039 & 0,53 \\
\hline \multicolumn{13}{|l|}{$\%$ domicílios } \\
\hline $\begin{array}{l}\text { com água } \\
\text { encanada }\end{array}$ & 0,94 & 0,000 & 0,99 & 0,94 & 0,000 & 0,99 & 0,94 & 0,000 & 0,99 & 0,78 & 0,487 & 0,99 \\
\hline$\%$ domicílios & & & & & & & & & & & & \\
\hline $\begin{array}{l}\text { com coleta de } \\
\text { lixo }\end{array}$ & 0,95 & 0,000 & 1,00 & 0,95 & 0,000 & 1,00 & 0,95 & 0,000 & 1,00 & 0,76 & 0,152 & 1,00 \\
\hline $\begin{array}{c}\% \text { esgotamento } \\
\text { inadequados }\end{array}$ & $-0,97$ & 0,000 & 0,99 & $-0,97$ & 0,000 & 0,99 & $-0,97$ & 0,000 & 0,99 & $-0,70$ & 0,102 & 0,99 \\
\hline IDHM & 0,95 & 0,000 & 0,99 & 0,94 & 0,000 & 0,99 & 0,94 & 0,000 & 0,99 & 0,77 & 0,040 & 0,99 \\
\hline $\begin{array}{c}\text { IDHM } \\
\text { Longevidade }\end{array}$ & 0,95 & 0,000 & 1,00 & 0,95 & 0,000 & 1,00 & 0,95 & 0,000 & 1,00 & 0,75 & 0,040 & 1,00 \\
\hline \multicolumn{13}{|l|}{ Saúde } \\
\hline $\begin{array}{c}\text { Taxa de } \\
\text { fecundidade } \\
\text { total }\end{array}$ & $-0,97$ & 0,000 & 0,99 & $-0,97$ & 0,000 & 0,99 & $-0,97$ & 0,000 & 0,99 & $-0,71$ & 0,045 & 0,99 \\
\hline $\begin{array}{l}\text { Esperança de } \\
\text { vida ao nascer }\end{array}$ & 0,96 & 0,000 & 0,99 & 0,95 & 0,000 & 0,99 & 0,95 & 0,000 & 0,99 & 0,75 & 0,498 & 0,99 \\
\hline $\begin{array}{l}\% \text { mulheres } \\
\text { jovens com } \\
\text { filhos }\end{array}$ & $-0,97$ & 0,000 & 1,00 & $-0,96$ & 0,000 & 1,00 & $-0,96$ & 0,000 & 1,00 & $-0,72$ & 0,050 & 1,00 \\
\hline $\begin{array}{c}\text { Mortalidade } \\
\text { infantil }\end{array}$ & $-0,99$ & 0,000 & 0,99 & $-0,98$ & 0,000 & 0,99 & $-0,98$ & 0,000 & 0,99 & $-0,66$ & 0,276 & 0,99 \\
\hline
\end{tabular}




\begin{tabular}{|c|c|c|c|c|c|c|c|c|c|c|c|c|}
\hline $\begin{array}{l}\text { Mortalidade até } \\
\qquad 5 \text { anos }\end{array}$ & $-0,99$ & 0,000 & 1,00 & $-0,99$ & 0,000 & 1,00 & $-0,99$ & 0,000 & 1,00 & $-0,64$ & 0,359 & 1,00 \\
\hline $\begin{array}{c}\text { Taxa de } \\
\text { envelhecimento }\end{array}$ & 0,95 & 0,000 & 0,95 & 0,94 & 0,000 & 0,95 & 0,95 & 0,000 & 0,95 & 0,76 & 0,069 & 0,95 \\
\hline $\begin{array}{l}\text { Probabilidade } \\
\text { de sobreviver } \\
\text { até } 60 \text { anos }\end{array}$ & 0,95 & 0,000 & 1,00 & 0,95 & 0,000 & 1,00 & 0,95 & 0,000 & 1,00 & 0,74 & 0,294 & 1,00 \\
\hline
\end{tabular}

Fonte: Elaborada pelos autores.

Legenda: $\mathrm{AB}=$ Atenção Básica, $\mathrm{ACS}=$ Agente Comunitário de Saúde; EqSF = Equipe Saúde da Família e NASF = Núcleos de Apoio

à Saúde da Família, IDHM= índice de desenvolvimento humano municipal, $r=$ Correlação e $P=$ valor-P.

Todas as variáveis de desenvolvimento e de saúde referentes ao estudo apresentaram modificação no coeficiente de correlação e de determinação, com significância estatística para a $\mathrm{AB}$ e para cada estratégia/programa específico, sendo que os relativos ao NASF apresentaram uma significância estatística apenas para três variáveis de desenvolvimento: Índice de Gini $(\mathrm{P}=0,039)$, IDHM $(\mathrm{P}=0,040)$ e IDHM de Longevidade $(\mathrm{P}=0,040)$ e para duas variáveis de saúde: taxa de fecundidade total $(\mathrm{P}=0,045)$ e a porcentagem de mulheres jovens com filhos $(\mathrm{P}=0,050)$ (Tabela 2$)$.

A mudança percentual média (APC) de ACS no período de 1998 a 2000 foi de $15,0 \%$, no período de 2000 a 2005 a APC caiu para 4,0\% e, no período de 2005 a 2016, essa mudança foi de apenas 1,1\%. A APC de EqSF de 1998 a 2001 foi de 64,5\% com diferença de 49,5\% em relação à APC de ACS no mesmo período; de 2001 a 2005 a APC de EqSF foi de 15,3\% com redução de 49,2\% comparado com o período de 1998 a 2001; já no período de 2005 a 2016 a APC de EqSF foi de apenas 2,8\% com redução de 61,7\% comparado ao período da tendência 1 (Tabela 3).

Tabela 3

Variáveis ACS e EqSF por tendência temporal de 1998 a 2016. Fortaleza, CE, 2017.

\begin{tabular}{|c|c|c|c|c|c|c|c|c|c|}
\hline \multirow{3}{*}{ VARIÁVEIS } & \multicolumn{6}{|c|}{ Tendências } & \multirow{3}{*}{$\begin{array}{l}\text { Total } \\
\text { AAPC }\end{array}$} & \multirow{2}{*}{\multicolumn{2}{|c|}{ IC $95 \%$}} \\
\hline & \multicolumn{3}{|c|}{ Tempo } & APC & \multicolumn{2}{|c|}{ IC 95\% } & & & \\
\hline & 1 & 1998 & 2000 & $15,0^{*}$ & 5,7 & 25,1 & & & \\
\hline \multirow[t]{3}{*}{ ACS } & 2 & 2000 & 2005 & $4,0^{*}$ & 1,3 & 6,8 & $3,4^{*}$ & 2,2 & 4,5 \\
\hline & 3 & 2005 & 2016 & $1,1^{*}$ & 0,5 & 1,7 & & & \\
\hline & 1 & 1998 & 2001 & $64,5^{*}$ & 54,5 & 75,3 & & & \\
\hline \multirow[t]{2}{*}{ EqSF } & 2 & 2001 & 2005 & $15,3^{*}$ & 8,3 & 22,9 & $14,1^{*}$ & 12,2 & 15,9 \\
\hline & 3 & 2005 & 2016 & $2,8^{*}$ & 1,9 & 3,7 & & & \\
\hline
\end{tabular}

Fonte: Elaborada pelos autores.

Nota: $A P C=$ mudança percentual média; AAPC = mudança percentual anual média; IC 95\% = intervalo de confiança de $95 \%$. População disponível apenas do CENSO 2010.

*Significantemente diferente de $0(p<0,05)$. 
Observa-se tendência de crescimento tanto para ACS quanto EqSF. Não foi possível calcular para NASF, pois sua implantação ocorreu há apenas sete anos. Para os ACS, foram detectadas três tendências no período de estudo (1998-2000; 2000-2005 e 2005-2016), sendo que a APC teve decrescimento de $9,9 \%$ (variando de $15 \%$ a $1,1 \%$ ). Para as EqSF, também foram detectadas três tendências no período de estudo (1998-2001; 2001-2005 e 2005-2016), variando de $64,5 \%$ a $2,8 \%$. A mudança percentual anual média no período de estudo para ACS foi de 3,4\% e para EqSF foi 14,1\% (Tabela 3, Gráficos 2 e 3).

\section{Gráfico 2}

Variação da mudança percentual média de ACS por períodos, de 1998-2001; 2000-2005 e 2005-2016. Fortaleza, CE, 2017.

All: 2 Joinpoints

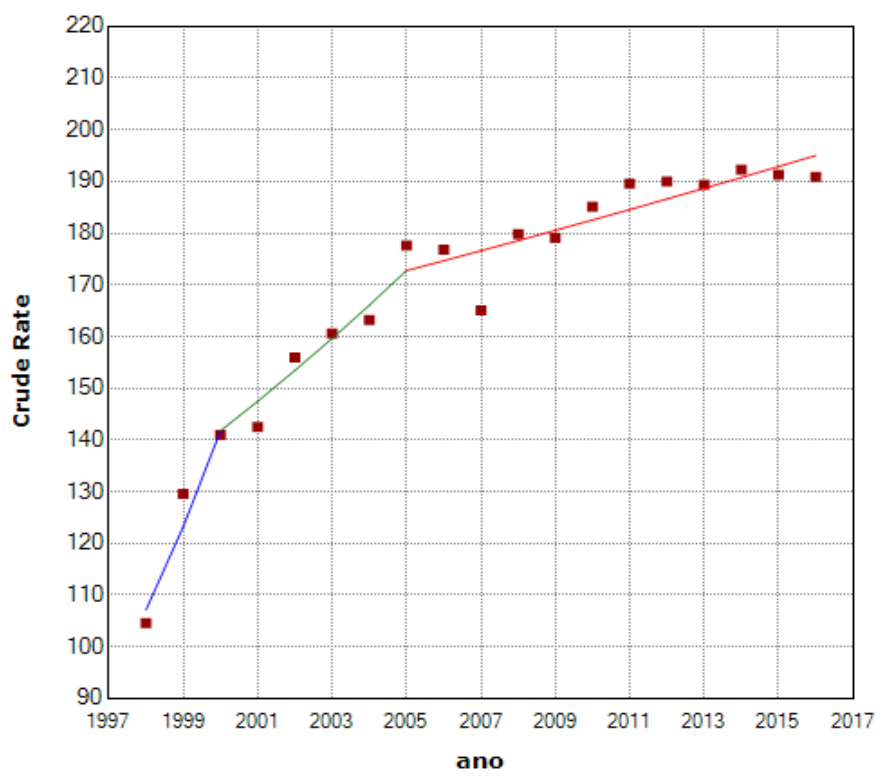


Variação da mudança percentual média de EqSF por períodos, de 1998-2001; 2001-2005 e 2005-2016. Fortaleza CE, 2017.

All: 2 Joinpoints
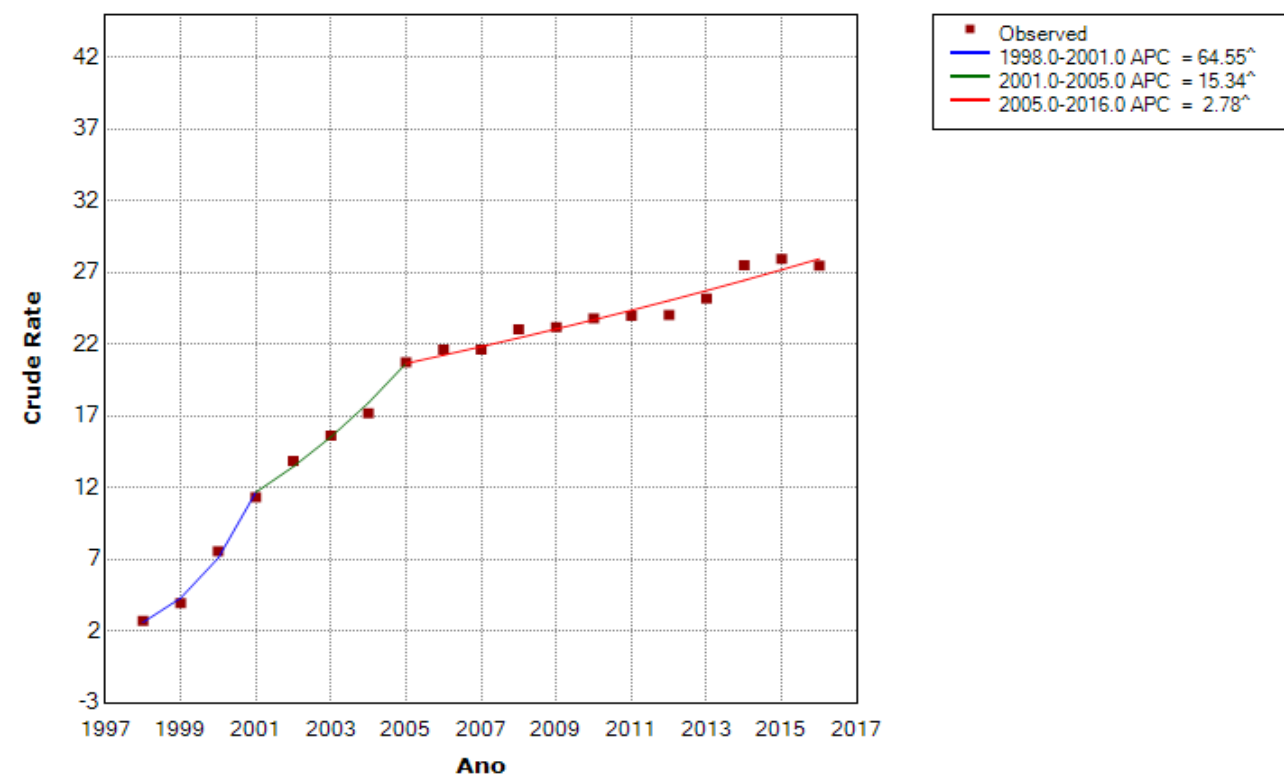

$\hat{\wedge}$ Indicates that the Annual Percent Change (APC) is significantly different from zero at the alpha $=0.05$ level Final Selected Model: 2 Joinpoints.

\section{Discussão}

Há uma tendência de crescimento na implantação da APS no Nordeste brasileiro, com destaque para ACS e EqSF, não sendo possível calcular a tendência do NASF, por sua recente implantação. Os indicadores de desenvolvimento e saúde apresentam correlação forte e positiva com a implantação da APS, tanto para ACS quanto EqSF, e de forma menos intensa para NASF.

Com o início do processo de consolidação da descentralização dos recursos na esfera do SUS, o PACS foi efetivamente instituído e regulamentado como estratégia de aprimoramento e consolidação da APS (Cunha e Campos, 2011). A criação dos ACS no Ceará teve sua origem inspirada pela experiência pioneira do Projeto Integrado de Saúde Comunitária desenvolvido pela Universidade de Brasília em Planaltina-DF. Na região Nordeste, diante de um período prolongado de seca em 1987, foi idealizada a atuação desses ACS, implantado oficialmente em 1988 com a contratação dos primeiros ACS na cidade de Jucás-CE (Ávila, 201 1; Brevidelli e Freitas, 201 1; Sumar e Fausto, 2014). 
Os ACS eram supervisionados por um profissional de enfermagem. Todavia, as demandas em saúde da população levaram gestores a incluírem o profissional médico nessa equipe, o que constituiu a EqSF e originou o PSF em uma cidade cearense - Quixadá (Campos, Faria e Santos, 2010; Ávila, 2011; Brevidelli e Freitas, 2011; Guedes, Santos e Lorenzo, 2011).

O movimento de implementação do PSF - que propunha oferecer cobertura universal, chegando inclusive às realidades mais distantes - foi intenso no Nordeste, especialmente com a descentralização político-administrativa do setor saúde. Assim, em 1994, o MS formatou o programa a nível nacional com o objetivo de mudar o modelo da assistência, priorizando a promoção da saúde e a prevenção de agravos. Dessa forma, de uma estratégia local, passou-se a um programa nacional com foco no fortalecimento da APS (Brasil, 2011; Guedes, Santos e Lorenzo, 2011; Arantes, Shimizu e MerchánHamann, 2016).

A grande expansão do PSF ocorreu depois de 1998, com ampliação quantitativa e geográfica da cobertura, devido ao aumento progressivo no número de municípios que aderiram ao programa em todas as regiões do país. Em 2006, o PSF, já implantado em vários municípios, deixou de ser compreendido apenas como iniciativa restrita e pontual e foi elevado ao patamar de estratégia para orientar a organização do SUS com base na APS. Em 2006, institui-se a ESF, modelo brasileiro prioritário para estruturação da APS (Guedes, Santos e Lorenzo, 2011; Arantes, Shimizu e Merchán-Hamann, 2016).

Um ponto a ser observado no plano de fundo da grande implantação das EqSFs é o processo de descentralização da gestão em saúde. Houve um reforço do processo de municipalização com os instrumentos normalizadores (NOBs) nos anos 1990 e os gestores municipais passaram a fortalecer as ações da APS, norteadas, à época, pelo PSF. Percebe-se, dessa forma, um incentivo e a criação de possibilidade para a ampliação do número de ACSs e de EqSFs (Scatena e Tanaka, 2001; Sumar e Fausto, 2014). O processo de implementação de ACSs e EqSFs na região Nordeste se deu de forma simultânea na maioria dos municípios como consequência da municipalização (Guedes, Santos e Lorenzo, 2011; Arantes, Shimizu e Merchán-Hamann, 2016).

No ano de 2002, na região Nordeste, $78 \%$ dos municípios já haviam aderido ao programa e existiam no país 155.847 ACSs e 13.661 EqSFs (Silva, 2002). Os dados deste estudo revelam que nesse mesmo período, a região Nordeste concentrava $48 \%$ e $49 \%$ do total do contingente destas modalidades a nível nacional (75.377 ACS e 6.699 EqSF). Esta intensa implantação decorre do fato que o Nordeste brasileiro, com suas muitas vulnerabilidades sociais e programáticas, estimulou e propiciou o movimento de implantação do PSF. A região Nordeste está entre as regiões onde essas deficiências são mais acentuadas, indicando a necessidade de priorizar políticas públicas que superem essas diferenças e garantam o princípio da universalidade da assistência à 
saúde do SUS (Brevidelli e Freitas, 2011; Mendes, 2015; Arantes, Shimizu e Merchán-Hamann 2016).

Neste estudo, observa-se uma disparidade entre o número de equipes credenciadas, cadastradas e implantadas, o que revela um hiato entre a percepção das necessidades locais, o planejamento dos gestores e a execução, de fato, das ações. Talvez possa ser percebida mudança nesse cenário com a publicação da portaria n. 2.436 de setembro de 2017 que reformula alguns aspectos da Política Nacional de Atenção Básica (PNAB) (Brasil, 2017) e propõe prazo de implantação das equipes, com definição de no máximo quatro meses depois da publicação do credenciamento (Brasil, 2011).

De acordo com a PNAB, o número de ACSs deve ser suficiente para cobrir $100 \%$ da população cadastrada, para tanto se recomenda o limite máximo de 750 pessoas por ACS e de 12 ACSs por EqSF. Cada EqSF deve ser responsável por no máximo 4.000 pessoas, e a média recomendada é de 3.000 (Brasil, 2017a). No período de análise deste estudo, o Nordeste brasileiro apresentou uma média de 607 habitantes por ACS; e média de 8.082 habitantes por EqSF. Isso significa um impacto negativo no processo de reorganização da APS desta região, uma vez que um dos itens necessários para sua implementação não está sendo respeitado.

Com o objetivo de apoiar a consolidação da APS no Brasil, o Ministério da Saúde (MS) cria, em 2008, os NASFs. Já no ano de 2010, surgem registros de implementação dos primeiros NASFs na região Nordeste. A criação dos NASFs é uma resposta às necessidades das EqSFs já atuantes a fim de agir na complexidade dos casos e nas demandas que exigiam a presença de outras categorias profissionais como retaguarda técnico-pedagógica. Os NASFs devem atuar na proposta do apoio matricial, o que significa um modo de realizar a atenção em saúde de forma compartilhada com vistas à integralidade e à resolubilidade da atenção, por meio do trabalho interprofissional (Sumar e Fausto 2014; Mendes, 2015; Arantes, Shimizu e Merchán-Hamann 2016; Assis et al., 2017; Mendonça et al., 2017).

Atualmente, o Ministério da Saúde financia e reconhece três modalidades de NASFs. Ao NASF 1 podem estar vinculadas de 5 a 9 EqSFs; ao NASF 2 podem estar vinculadas, no mínimo de 3 e no máximo de 4 EqSFs. Para o NASF 3, deverá ser vinculada de 1 a 2 EqSFs (Mendes, 2015; Mendonça et al., 2017).

Logo da criação dos NASFs, percebe-se um aumento expressivo na sua quantidade. Observa-se, todavia, um segundo 'surto' de crescimento no número de NASFs desde 2012, com a criação do NASF 3. Com o surgimento da modalidade 3 de NASF, com base na portaria n. 3.124, ocorreu a possibilidade para implantação do NASF nos municípios de pequeno porte - o que causou um boom no processo de expansão das equipes de retaguarda. Os dados deste estudo corroboram com essa cronologia, quando mostram que em 2013 a região Nordeste apresentou aumento significativo no número de NASFs. Vale 
ainda ressaltar que, mesmo com esse crescimento expressivo, este estudo observou que a região Nordeste apresenta um déficit no número de equipes de retaguarda para o total das EqSFs existentes.

Os resultados deste estudo não possibilitam ver a real distribuição de EqSFs por equipe NASF, mas estima-se que há uma sobrecarga nessa cobertura. A grande demanda acarreta fragilidades ao trabalho dos profissionais do NASF. A deficiência de recursos de saúde é preocupante, pois na maioria das regiões do país não há recursos para financiar uma infraestrutura de saúde adequada à prestação de assistência mais complexa e especializada e com profissionais de saúde qualificados (Brevidelli e Freitas, 2011; Arantes; Shimizu; Merchán-Hamann 2016).

A implantação desigual da $\mathrm{AB}$ nos estados do Nordeste brasileiro pode estar associada à disponibilidade financeira dos municípios e, em alguns casos, à disponibilidade de profissionais que compõem essas equipes, especialmente os profissionais médicos, que são obrigatórios para compor a EqSF. Quando não há médico na equipe, esta é considerada como equipe do PACS, o que altera os quantitativos apresentados pelo DAB. Acredita-se que em razão desse desafiador provimento de profissionais, houve mudança na PNAB em 2012, flexibilizando a carga horária dos médicos para que as equipes não deixem de estar regulamentadas pela falta desse profissional. Isso acontece especialmente nos municípios de pequeno porte e distante dos grandes centros, onde essa fixação é difícil. Mediante esse cenário, incita-se a reflexão de que as políticas públicas devem ir além do incremento da infraestrutura em saúde, mas precisa também promover a viabilidade e, sobretudo, a qualificação dos profissionais de saúde para o desenvolvimento das competências necessárias para a atuação no modelo da APS (Brevidelli e Freitas, 2011; Sumar e Fausto, 2014; Mendes, 2015).

Recursos básicos escassos e pouco qualificados acarretam prejuízos no índice de cobertura e revelam um déficit considerável em condições básicas de vida e no acesso a programas de atenção primária (Brevidelli e Freitas, 2011; Arantes, Shimizu e Merchán-Hamann, 2016). Contudo, apesar dos investimentos e estímulos, grande parte do território nacional ainda está sujeita a essa realidade de provimento da força de trabalho em saúde. Os menores valores de investimentos de recursos na saúde foram encontrados em alguns estados do Nordeste, a seguir por ordem decrescente do valor: Maranhão, Sergipe e Alagoas (Brevidelli e Freitas, 2011).

A efetividade da APS pode ser medida através das Hospitalizações por Condições Sensíveis ao Cuidado Primário. Um estudo recente verificou que os indicadores de acesso a APS e a vulnerabilidade social têm efeito sobre a taxa de hospitalização. De forma que, a cada ponto a mais no índice de acesso à APS, ocorria redução de oito hospitalizações por condições sensíveis à APS. Em relação ao risco socioeconômico, quanto maior a população de alto 
risco, maior será o índice de hospitalização. Tal estudo evidencia os impactos da qualidade da APS e diferentes determinantes no processo saúde-doença nas taxas de hospitalização, mostrando que, no Brasil, há uma parcela significativa da população sem acesso à APS, com histórico grave e desafiador de questões como desigualdade de renda e baixa escolarização (Arantes, Shimizu e Merchán-Hamann 2016; Mendonça et al., 2017).

As ações da APS podem reduzir as internações hospitalares tanto por doenças infecciosas, através da imunização específica, quanto por complicações agudas das doenças crônicas não-transmissíveis, através do controle da hipertensão, diabetes e doenças cardíacas (Assis et al., 2017). Essas estratégias foram incluídas na lista de ações da ESF (Dourado et al., 2011).

A dimensão da efetividade da APS mostra-se altamente desenvolvida em todo território nacional, o que significa melhores condições de vida e sobrevida de recém-nascidos e crianças menores de 1 ano, além de maior capacidade da população em prevenir doenças infectocontagiosas e parasitárias. Esse avanço pode ser atribuído também ao crescente desenvolvimento socioeconômico e educacional brasileiro observado nas últimas décadas. Contudo, também foram evidenciadas desigualdades regionais, ficando a maioria dos estados do Norte/Nordeste em níveis inferiores aos do Sul/Sudeste ao se falar sobre a efetividade da APS. Paradoxalmente, o Nordeste brasileiro, que no período de 1999 a 2007 estava em crescente processo de implementação da APS, atingindo, no ano de 2007, 100\% de cobertura, nesse mesmo período, ocupava o segundo lugar nas internações hospitalares sensíveis à atenção básica por região do Brasil (Dourado et al., 2011).

Assim, este estudo observou que a implementação da APS no Nordeste brasileiro ocorreu de forma intensa, mas o êxito das suas ações não necessariamente acompanhou seu processo de expansão, ainda existindo alguns desafios à plena consolidação desse modelo. O que pode levar a acreditar que a correlação forte entre os diversos indicadores sociais estudados e a implantação da APS pode também ter correlação com outros aspectos não abordados nesse estudo, aqui considerados como potenciais fatores de confusão da efetividade da $\mathrm{AB}$. Não se pode precisar exatamente quais seriam esses fatores de confusão, mas é notório que o país, ao longo do período delimitado para esse estudo, passou por diversas mudanças sociais, econômicas e culturais que podem ter interferido nos indicadores sociais e de saúde.

Uma das limitações apresentadas por esta pesquisa diz respeito ao desenho de estudo ecológico, tendo em vista que, ao se analisar as variáveis em âmbito regional, elas não representam necessariamente uma associação a nível estadual. Ademais, a coleta de dados provenientes de registros de sistemas de informação pode traduzir uma realidade pouco fidedigna ao se levar em consideração os sub-registros dos dados relacionados às variáveis aqui analisadas. 


\section{Considerações Finais}

O presente estudo verificou que há uma tendência temporal de crescimento na implantação da APS no Nordeste brasileiro, com destaque para ACS e EqSF, bem como a existência de forte correlação entre os indicadores sociais e de saúde com a implantação da $\mathrm{AB}$.

Tornam-se necessários estudos in loco que investiguem os potenciais fatores de confusão entre indicadores sociais e de saúde e a efetividade da APS, bem como estudos com unidade de análise a nível municipal, uma vez que são os municípios que estão diretamente em conformidade com o modelo descentralizado do Sistema Nacional de Saúde do Brasil, sendo ele o local direto para a implementação das Políticas Públicas em Saúde.

\section{MODIFICACIONES EN LOS INDICADORES SOCIALES DE LA REGIÓN NORDESTE DESPUÉS DE LA IMPLEMENTACIÓN DE LA ATENCIÓN PRIMARIA}

Resumen El objetivo fue describir la implementación de la Atención Primaria de la Salud en el Nordeste brasileño y su correlación con los indicadores sociales. Se trata de un estudio ecológico con datos del Departamento de Atención Básica del Ministerio de Salud de Brasil y del Instituto Brasileño de Geografía y Estadística, tomando como variable de estudio el número de Agentes Comunitarios de Salud, Equipos de Salud de la Familia y Núcleos de Apoyo a la Salud de la Familia. El cálculo de la tendencia temporal de Agentes Comunitarios de Salud y Equipos de Salud de la Familia fue realizado por la regresión joinpoint de Poisson. Se observó una fuerte correlación $(r \geq 0,7)$ entre los indicadores sociales y la implantación de la Atención Primaria de la Salud y del Núcleo de Salud de la Familia en la región Nordeste, alcantarillado sanitario inadecuado, tasa de fecundidad general, domicilio con agua entubada, porcentaje de domicilios con servicio de recolección de residuos, esperanza de vida al nacer, tasa de envejecimiento y probabilidad de sobrevivir hasta los 60 años. Las variables de desarrollo y salud presentaron modificación en el coeficiente de correlación y de determinación, con significación estadística $(\mathrm{p}<0,05)$. Se observa una tendencia temporal de crecimiento en la implantación de la Atención Primaria de la Salud en el Nordeste, así como la existencia de una fuerte correlación entre los indicadores sociales y de salud.

Palabras clave evaluación en salud; Atención Primaria de la Salud; Estrategia de Salud de la Familia; indicadores sociales; salud pública. 


\section{Colaboradores}

Fátima Cristina Dias de Carvalho responsabilizou-se pela concepção do projeto de pesquisa, análise e interpretação dos dados, redação e revisão crítica; Thiago Brasileiro de Vasconcelos fez análise e interpretação dos dados, redação e revisão crítica; Gisele Maria Melo Soares Arruda fez análise e interpretação dos dados, redação e revisão crítica; Raimunda Hermelinda Maia Macena responsabilizou-se pela concepção do projeto de pesquisa, análise e interpretação dos dados, redação e revisão crítica.

\section{Financiamento}

Conselho Nacional de Desenvolvimento Científico e Tecnológico (CNPq) por meio de bolsas de Iniciação Científica.

\section{Notas}

${ }^{1}$ Disponível em: http://dab.saude.gov.br/portaldab/historico_cobertura_sf.php [acesso em 07/12/2018]

${ }^{2}$ Disponível em https://www.ibge.gov.br/estatisticas-novoportal/sociais/populacao/9103 estimativas - de-populacao.html [acesso em 07/12/2018]

${ }^{3} \mathrm{O}$ universo de indivíduos é limitado àqueles que vivem em domicílios particulares permanentes.

${ }^{4}$ A água pode ser proveniente de rede geral, de poço, de nascente ou de reservatório abastecido por água das chuvas ou carro-pipa.

${ }^{5}$ Estão incluídas as situações em que a coleta de lixo realizada diretamente por empresa pública ou privada, ou o lixo é depositado em caçamba, tanque ou depósito fora do domicílio, para posterior coleta pela prestadora do serviço.

${ }^{6}$ Através da fórmula: [(valor observado do indicador) - (valor mínimo)] / [(valor máximo) (valor mínimo)], em que os valores mínimos e máximos são 25 e 85 anos, respectivamente.

\section{Referências}

AKERMAN, Marco; FURTADO, Juarez P. Práticas de avaliação em saúde no Brasil: diálogos. Porto Alegre: Rede Unida, 2016. 367 p. Disponível em: <http://historico. redeunida.org.br/editora/biblioteca-digital/ serie-atencao-basica-e-educacao-na-saude/ praticas-de-avaliacao-em-saude-no-brasildialogos-pdf>. Acesso em: 10 abr. 2017.

ARANTES, Luciano J.; SHIMIZU, Helena E.; MERCHÁN-HAMANN, Edgar. Contribuições e desafios da Estratégia Saúde da Família na 
Atenção Primária à Saúde no Brasil: revisão da literatura. Ciência \& Saúde Coletiva, Rio de Janeiro, v. 5, n. 21, p. 1.499-1.509, 2016.

ASSIS, Juvêncio C. L. et al. A vivência de um fisioterapeuta em uma Residência Multiprofissional: desafios e possibilidades. Revista APS, Juiz de Fora, v. 20, n. 2, p. 279-287, 2017.

ÁVILA, Maria M.M. A case study of the Community Health Agents Program in Uruburetama, Ceará (Brazil). Ciência \& Saúde Coletiva, Rio de Janeiro, v. 16, n. 1, p. 349-360, 2011.

BRASIL. Portaria $n^{\circ} 2.488$, de 21 de outubro de 2011. Política Nacional de Atenção Básica. Brasília, DF, 21 out. 2011. Disponível em: <http://bvsms.saude.gov.br/bvs/saudelegis/ gm/2011/prt2488_21_10_2011.html > . Acesso em: 25 abr. 2017.

BRASIL. Resolução $n^{0}$ 510, de 07 de Abril de 2016. Brasília, DF, 24 maio 2017. Seção 01, p. 44-46. Disponível em: <http://conselho. saude.gov.br/resolucoes/2016/reso510.pdf $>$. Acesso em: 20 set. 2017.

BRASIL. Departamento de Atenção Básica. Ministério da Saúde. Quais as mudanças na atualização da PNAB? 2017. Disponível em: $<$ http://dab.saude.gov.br/portaldab/noticias. php . conteudo=_\&cod $=2433>$. Acesso em: 4 ago. 2017.

BREVIDELLI, Maria M.; FREITAS, Fernando C. G.. Estudo ecológico sobre o desenvolvimento da saúde no Brasil. Ciência \& Saúde Coletiva, Rio de Janeiro, v. 9, n. 17, p. 2.471-2.480, 2011.

CAMPOS, Francisco C. C.; FARIA, Horácio P.; SANTOS, Max A. Planejamento e avaliação das ações em saúde. 2. ed. Belo Horizonte: Nescon/UFMG, 2010.

CUNHA, Gustavo T.; CAMPOS, Gastão W. S. Apoio matricial e atenção primária em saúde. Saúde e Sociedade, São Paulo, v. 20, n. 4, p. 961-970, 2011.
DOURADO, Ines et al. Trends in Primary Health Care-sensitive Conditions in Brazil. Medical Care, Massachusetts, p. 577-584. Jun. 2011.

FIGUEIREDO, Elisabeth N. A Estratégia Saúde da Família na Atenção Básica do SUS. Brasília: Unifesp, 2012. 10 p. Disponível em: <https:// www.unasus.unifesp.br/biblioteca_virtual/esf/3/ unidades_conteudos/unidade06/unidade06. pdf > . Acesso em: 15 abr. 2017.

FRANCISCO, Wagner C. População do Nordeste. Brasil Escola. Disponível em: <http://brasilescola. uol.com.br/brasil/populacao-nordeste.htm>. Acesso em: 30 nov. 2017.

GIOVANELLA, Lígia; MENDONÇA, Maria H. M. Atenção Primária à Saúde: seletiva ou coordenadora dos cuidados? Rio de Janeiro: CEBES, 2012.

GUEDES, Jose S.; SANTOS, Rosa M. B. ; DI LORENZO, Rosicler A. V. A Implantação do Programa de Saúde da Família (PSF) no Estado de São Paulo (1995-2002). Saúde e Sociedade, São Paulo, v. 4, n. 20, p. 875-883, 2011.

MALTA, Deborah C. et al. A cobertura da Estratégia de Saúde da Família (ESF) no Brasil, segundo a Pesquisa Nacional de Saúde. Ciência \& Saúde Coletiva, Rio de Janeiro, v. 21, n. 2, p. 327-338, 2016.

MATTA, Gustavo C.; MOROSINI, Márcia V. G. Atenção primária à saúde. 2009. Escola Politécnica de Saúde Joaquim Venâncio. Rio de Janeiro. Disponível em:<http://www. sites.epsjv.fiocruz.br/dicionario/verbetes/ ateprisau.html>. Acesso em: 3 jun. 2017.

MENDES, Eugênio V. A construção social da atenção primária à saúde. Brasília: CONASS, 2015.

MENDES, Eugênio V. As redes de atenção à saúde. 2. ed. Brasília: Organização Pan-americana da Saúde, 2011. 554 p. Disponível em: <http:// www.paho.org/bra/index.php?option $=$ com docman \& view = download \& category slug=servicos-saude-095\&alias=1402-as-redes- 
atencao-a-saude-2a-edicao-2\&Itemid $=965>$. Acesso em: 15 abr. 2017.

MENDONÇA, Claunara S. et al. Hospitalizations for primary care sensitive conditions: association with socioeconomic status and quality of family health teams in Belo Horizonte, Brazil. Health Policy And Planning, London, v. 10, n. 32, p. 1.368-1.374, 2017.

MORETTI, Patrícia G. S.; FEDOSSE, Elenir. Núcleos de Apoio à Saúde da Família: impactos nas internações por causas sensíveis à atenção básica. Fisioterapia e Pesquisa, São Paulo, v. 23, n. 3, p. 241-247, set. 2016.

OLIVEIRA, Maria A. de C.; PEREIRA, Iara C. Atributos essenciais da Atenção Primária e a Estratégia Saúde da Família. Revista Brasileira de Enfermagem, Brasília, n. 66, p.158-164, 2013.
SCATENA, João H. G.; TANAKA, Oswaldo Y. Os instrumentos normalizadores (NOB) no processo de descentralização da saúde. Saúde e Sociedade: Revista USP, São Paulo, p. $47-74,2001$.

SILVA, Lígia M. V. et al. (Coord.). O programa de saúde da família: evolução de sua implantação no Brasil. Brasília: Ministério da Saúde, 2002. 70 p. Disponível em: <http://189.28.128.100/dab/ docs/geral/psf_evolucao_brasil.pdf $>$. Acesso em: 20 mar. 2017. Coordenação: Lígia Maria Vieira da Silva, Zulmira M. Araújo Hartz

SUMAR, Norhan; FAUSTO, Márcia C. R. Atenção Primária à Saúde: a construção de um conceito ampliado. Journal of Management \& Primary Health Care, São Paulo, v. 5, n. 2, p. 202-212, 2014. 